Virginia Commonwealth University VCU Scholars Compass

2006

\title{
Electronic counting rules for the stability of metal- silicon clusters
}

\author{
J. Ulises Reveles \\ Virginia Commonwealth University \\ Shiv N. Khanna \\ Virginia Commonwealth University, snkhanna@vcu.edu
}

Follow this and additional works at: http://scholarscompass.vcu.edu/phys_pubs

Part of the Physics Commons

Reveles, J.U., Khanna, S.N. Electronic counting rules for the stability of metal-silicon clusters. Physical Review B, 74, 035435 (2006). Copyright (C) 2006 American Physical Society.

\section{Downloaded from}

http://scholarscompass.vcu.edu/phys_pubs/80

This Article is brought to you for free and open access by the Dept. of Physics at VCU Scholars Compass. It has been accepted for inclusion in Physics Publications by an authorized administrator of VCU Scholars Compass. For more information, please contact libcompass@vcu.edu. 


\title{
Electronic counting rules for the stability of metal-silicon clusters
}

\author{
J. Ulises Reveles and S. N. Khanna \\ Physics Department, Virginia Commonwealth University, Richmond, Virginia 23284-2000, USA \\ (Received 7 April 2006; revised manuscript received 26 May 2006; published 28 July 2006)
}

\begin{abstract}
First principles electronic structure calculations have been carried out to examine the stability of cationic, neutral, and anionic $M \mathrm{Si}_{15}, M \mathrm{Si}_{16}$, and $M \mathrm{Si}_{17}(M=\mathrm{Sc}$, Ti, and $\mathrm{V})$ clusters. $\mathrm{ScSi}_{16}{ }^{-}$, $\mathrm{TiSi}_{16}$, and $\mathrm{VSi}_{16}{ }^{+}$are found to be particularly stable in agreement with recent experiments. It is shown that the enhanced stability can be reconciled within a model where each $\mathrm{Si}$ atom coordinated to the metal contributes one electron to the valence pool. Clusters where the total number of valence electrons obtained by summing one electron from each Si site coordinated to metal atom and the valence electrons of the metal attain 20 are found to be particularly stable. Combined with the earlier reported stability at 18 electrons, it is proposed that such valence pools might be looked upon as a nearly free electron gas inside a silicon cage.
\end{abstract}

DOI: 10.1103/PhysRevB.74.035435

Silicon is a key element in the microelectronics industry and consequently, silicon clusters have attracted considerable attention in recent years. The interest is driven by the constant miniaturization of the electronic devices that motivates studies of behavior at nanometer scales as well as by the findings that the band gaps, stability, and local coordination in reduced sizes can be very different from those in bulk and change with size. Such an atomic control on the properties is vital for designing materials with tailored properties using cluster building blocks. ${ }^{1-3}$ While the unique behaviors in reduced sizes are encouraging, pure silicon clusters are highly reactive due to surface dangling bonds. ${ }^{4}$ Any efforts towards developing cluster based materials have to first address the stability and inertness of clusters. Finding simple rules that can guide the search for stable silicon based species is thus an important undertaking.

It was earlier proposed that silicon clusters can be stabilized by introducing endohedral metal atoms. ${ }^{5}$ Experiments ${ }^{6}$ and numerous theoretical studies ${ }^{2,3}$ indicate that even a single metal atom can change the reactivity. Earlier, Hiura et $a .^{6}{ }^{6}$ reported generating metal $(M)$-containing hydrogenated silicon clusters $\left(M \mathrm{Si}_{n} \mathrm{H}_{m}{ }^{+}\right)$by reacting transition metal ions $M^{+}$with $\mathrm{SiH}_{4}$. A comparison of the abundances in the mass spectra of $M \mathrm{Si}_{n}{ }^{+}$among $\left(M \mathrm{Si}_{n} \mathrm{H}_{m}{ }^{+}\right)$clusters, led them to propose that a $\mathrm{WSi}_{12}$ was a very stable species and that its stability could be reconciled within an 18 electron rule. ${ }^{7}$ Assuming that each $\mathrm{Si}$ contributes one electron to the valence manifold and that a W atom has 6 valence electrons, Hiura et al. argued that a total of 18 valence electrons would result in a closed electronic shell as in the case of rare gas atoms. Theoretical investigations revealed that the ground state of the cluster was a regular hexagonal prism of $12 \mathrm{Si}$ atoms with a $\mathrm{W}$ at the center. The compact geometrical shape and the accompanying electronic shell closure had similarities to the work in metal clusters where the electronic stability is sometimes reconciled within a jellium picture. ${ }^{8}$ Indeed, such as the octet rule used for rare gas atoms, a shell filling at 18 electrons occurs in a jellium model. ${ }^{8}$ This initial proposition, generated considerable excitement and Khanna et al. ${ }^{2}$ examined the 18-electron rule in $\mathrm{CrSi}_{n}(n=11-14)$ clusters since $\mathrm{Cr}$ also has six valence electrons such as W. They showed that the gain in binding energy in adding a $\mathrm{Si}$ atom to the $\mathrm{CrSi}_{n-1}$ was indeed large for $\mathrm{CrSi}_{11}$. This along with vertical
PACS number(s): 73.22.-f, 75.50.Pp

detachment energy (VDE) and the adiabatic electron affinity (AEA) supported the proposition. In an attempt to systematically examine the octet rule or the jellium picture, Reveles and Khanna ${ }^{9}$ recently examined the binding of transition metal atoms to $\mathrm{Si}_{12}$ across the entire $3 d$ transition metal series. Such a study allows investigations of stability as the number of valence electrons changes by one electron at a time. They demonstrated that $\mathrm{CrSi}_{12}$ indeed had the highest binding energy of all the $3 d$ elements. In addition to $\mathrm{Si}_{12}$ based clusters, Kumar and Kawazoe ${ }^{3}$ carried out first principles investigations on selected $M \mathrm{Si}_{16}$ clusters and proposed $\mathrm{TiSi}_{16}$ as another particularly stable species.

In the ground state structure of $\mathrm{CrSi}_{12}$, each $\mathrm{Si}$ is bonded to three other surface $\mathrm{Si}$ atoms and the $\mathrm{Cr}$ atom at the center. If the electronic manifold could be decomposed into $\mathrm{Si}-\mathrm{Si}$ bonds and silicon-metal manifolds, Reveles and Khanna suggested that the system may be looked upon as an outside silicon cage encapsulating a nearly free electron gas formed by selected silicon electrons and valence electrons of the metal. In its simplest form, the properties of the nearly free electron gas can be calculated within a jellium model. The resulting electronic spectrum exhibits a shell closing at 18 electrons and hence could account for the observed effect. The application of a jellium picture, however, does have ramifications since it also leads to a shell closing at 20 electrons. As Reveles and Khanna demonstrated, the calculated binding energy along the $3 d$ series did show a secondary peak at this number. The shell closing at 20 electrons is also consistent with the previously reported ${ }^{10}$ stability of $\mathrm{ZrSi}_{16}$ and also could account for some of the recent observations by Koyasu et al. ${ }^{11}$ Changing the metal atom to control the number of electrons also modifies the nuclear potential and hence, a more rigorous approach to isolate effects of electron counting, would be to vary the charged state of the cluster. This has been accomplished by Koyasu et al. ${ }^{11}$ These authors generated $M \mathrm{Si}_{16}(M=\mathrm{Sc}$, Ti, and $\mathrm{V})$ clusters via dual laser vaporization of a pure metal and silicon and studied cationic, neutral, and anionic clusters for all the species. Their investigations covered three important aspects. (1) Stability as seen through the mass spectra. (2) Negative ion photodetachment spectroscopy to probe the electronic states and to provide direct signature to the shell closings. (3) Reactivity studies with water molecules to examine the effect of open or closed electron shells on chemical activity. Noting that Sc, 
$\mathrm{Ti}$, and $\mathrm{V}$ contribute 3,4 , and 5 valence electrons respectively, these studies provide the most comprehensive work to verify the electron counting rules. The mass spectra indicate enhanced intensities at $\mathrm{ScSi}_{16}{ }^{-}, \mathrm{TiSi}_{16}$, and $\mathrm{VSi}_{16}{ }^{+}$clusters each of whom contain 20 valence electrons (assuming that each $\mathrm{Si}$ contributes one valence electron). Further $\mathrm{ScSi}_{16}{ }^{-}$ with 20 valence electrons had a vertical detachment energy (VDE) of $4.25 \mathrm{eV}$ and an adiabatic electron affinity (AEA) of $3.41 \mathrm{eV}$ that were considerably higher than other metals signifying a closed shell. Further, $\mathrm{VSi}_{16}$ with 21 valence electrons had the lowest ionization potential consistent with one extra electron beyond the closed shell. Reactivity studies also indicated a low reactivity for $\mathrm{ScSi}_{16}{ }^{-}, \mathrm{TiSi}_{16}$, and $\mathrm{VSi}_{16}{ }^{+}$ clusters again pointing to their closed electronic shells.

While the jellium picture and the experimental studies point to a 20 electron rule, a detailed first principles study is needed to clearly understand the experimental findings and to provide a more microscopic basis for such guiding rules. To illustrate the problem, if one assumes that each Si contributes one electron, $\mathrm{ScSi}_{17}, \mathrm{TiSi}_{17}{ }^{+}$, and $\mathrm{VSi}_{15}$ each have 20 electrons. However, none of these are seen as magic species in the observed mass spectra. Thus, the supposition of each Si contributing one valence electron to the nearly free electron gas needs further scrutiny. The purpose of the current paper is to accomplish this by carrying out first principles electronic structure calculations on neutral, cationic, and anionic $M \mathrm{Si}_{15}, M \mathrm{Si}_{16}$, and $M \mathrm{Si}_{17}(M=\mathrm{Sc}, \mathrm{Ti}$, and $\mathrm{V})$ clusters. In particular, we provide a microscopic basis for the 20 electron rule and outline the conditions under which a Si site can be regarded to donate an electron. Our objective goes beyond the 20 electron rule as we focus on the structural and electronic changes that mark the clusters when the electron count deviates from the shell closure. The theoretical results are compared with experimental findings to establish the validity of some of the rules.

The theoretical studies are carried out within a density functional formalism. ${ }^{12}$ The exchange and correlation effects are included using the generalized gradient approximation (GGA) of Perdew, Burke, and Ernzerhof. ${ }^{13}$ The electronic structure was determined using a linear combination of atomic orbitals molecular orbital approach. The wave function for the cluster is constructed by a linear combination of Gaussian type orbitals centered at the atomic positions in the cluster. The actual calculations employed two different numerical programs. Most of the studies were carried out using the Naval Research Laboratory Molecular Orbital Library (NRLMOL) set of codes developed by Pederson et al. ${ }^{14-16}$ For these calculations, we used a $6 s, 5 p$, and $3 d$ basis set for the $\mathrm{Si}$ atoms and $7 s, 5 p$, and $4 d$ basis for transition metal atoms. ${ }^{15}$ In each case, the basis set was supplemented by a diffuse Gaussian. For details, the reader is referred to the original papers. ${ }^{14-16}$ We also carried out supplementary calculations using deMon2K software ${ }^{17}$ in order to eliminate any uncertainties associated with the choice of basis set or the numerical procedure. In these studies, we employed a gradient corrected density functional ${ }^{13}$ and the double zeta valence polarized (DZVP) basis sets. ${ }^{18}$ To determine the ground state, the configuration space was sampled by starting from several initial configurations and optimizing the geometry by moving atoms in the direction of forces till they dropped below a threshold value. Since transition metal atoms are marked by nonzero spin multiplicities, the ground state determination included investigation over spin multiplicities. To analyze stabilities towards growth by addition of $\mathrm{Si}$ atoms, the calculations were conducted on cationic, neutral, and anionic $M \mathrm{Si}_{15}, M \mathrm{Si}_{16}$, and $M \mathrm{Si}_{17}(M=\mathrm{Sc}, \mathrm{Ti}$, and $\mathrm{V})$ clusters. The geometries were optimized without any symmetry constraint using Cartesian coordinates with the conjugate gradient method in the NRLMOL set of codes, and delocalized internal coordinates with the rational function optimization (RFO) and the Broyden, Fletcher, Goldfarb, and Shanno (BFGS) update in the deMon2K program. ${ }^{19}$

We start by considering the geometries of $M \mathrm{Si}_{15}, \mathrm{MSi}_{16}$, and $\mathrm{MSi}_{17}$ clusters. Kawamura and co-workers have earlier carried out extensive investigations on the $\mathrm{TiSi}_{n}, \mathrm{ZrSi}_{n}$, and $\mathrm{HfSi}_{n}$ clusters containing 8-16 Si atoms. ${ }^{20}$ In particular for $M \mathrm{Si}_{16}$ clusters, they found that the ground states were marked by fullerene like and Frank-Kasper like polyhedrons. Our studies included the geometries investigated by Kawamura and additional possibilities. For $\mathrm{TiSi}_{n}$ clusters, our findings are similar to those of Kawamura et al. However, the main focus of the current work is the variations in stability and electronic features as one changes the number of electrons either by changing the charged state or by changing the atomic number. Consequently, we also investigated cationic, neutral, and anionic species. Figure 1(a) shows the ground state geometries (bond lengths are labeled in $\AA$ ) for cationic, neutral, and anionic $\mathrm{ScSi}_{15}, \mathrm{TiSi}_{15}$, and $\mathrm{VSi}_{15}$ clusters. We also report in Fig. 1(a) the atomization energies (AE) and number of valence electrons $\left(N_{e}\right)$ for each cluster (the rational for determining $N_{e}$ would be discussed later). The AE was calculated using the expression

$$
\mathrm{AE}=n E(\mathrm{Si})+E(M)-E\left(M \mathrm{Si}_{n}\right)
$$

for the neutral species. For charged species, Eq. (1) was modified and the lower value between breaking into neutral Si and charged $M$ atom or one charged Si and neutral $M$ was taken. As pointed out in an earlier paper, a proper calculation of binding energy should include conservation of the spin degree of freedom. ${ }^{9,22}$ To this end, the calculation in Eq. (1) included the total energy of a state with the spin multiplicity for the $M$ or the Si atom that satisfies the Wigner-Witmer spin-conservation rule. ${ }^{9,22}$ In general the spin conservation was fulfilled with the use of ground state energies, however in some cases we needed to use excited states energies. As an example, in the case of $\mathrm{Si}_{15} \mathrm{~V}^{+}$cluster, the atomization energy was calculated using the expression:

$$
\mathrm{AE}=15 E\left({ }^{3} \mathrm{Si}\right)+E\left({ }^{3} \mathrm{~V}^{+}\right)-E\left({ }^{1} \mathrm{Si}_{15} \mathrm{~V}^{+}\right) .
$$

Here the superscripts indicate the spin multiplicity. In this equation we used the triplet excited state for the cationic $\mathrm{V}$ atom ${ }^{3} \mathrm{~V}^{+}$and not the quintet ground state ${ }^{5} \mathrm{~V}^{+}$. As pointed out earlier, ignoring the spin conservation rule may lead to erroneous conclusions. ${ }^{21}$ The corresponding geometries, $\mathrm{AE}$ and $N_{e}$ for $M \mathrm{Si}_{16}$ and $M \mathrm{Si}_{17}(\mathrm{M}=\mathrm{Sc}, \mathrm{Ti}$, and $\mathrm{V})$ clusters are shown in Figs. 1(b) and 1(c), respectively. In all cases, the clusters were found to have the lowest spin multiplicity consistent with the number of electrons. It is interesting to note that a calculation of the $\mathrm{AE}$ without enforcing the spin con- 


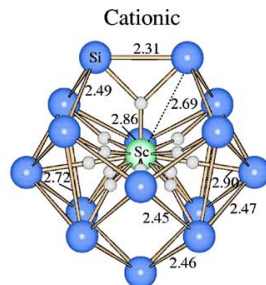

$\mathrm{AE}=60.75, \mathrm{~N}_{\mathrm{e}}=16$

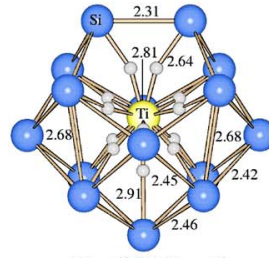

$\mathrm{AE}=63.27, \mathrm{~N}_{\mathrm{e}}=16$

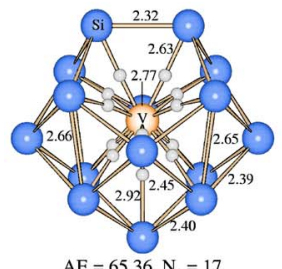

$\mathrm{AE}=65.36, \mathrm{~N}_{\mathrm{e}}=17$
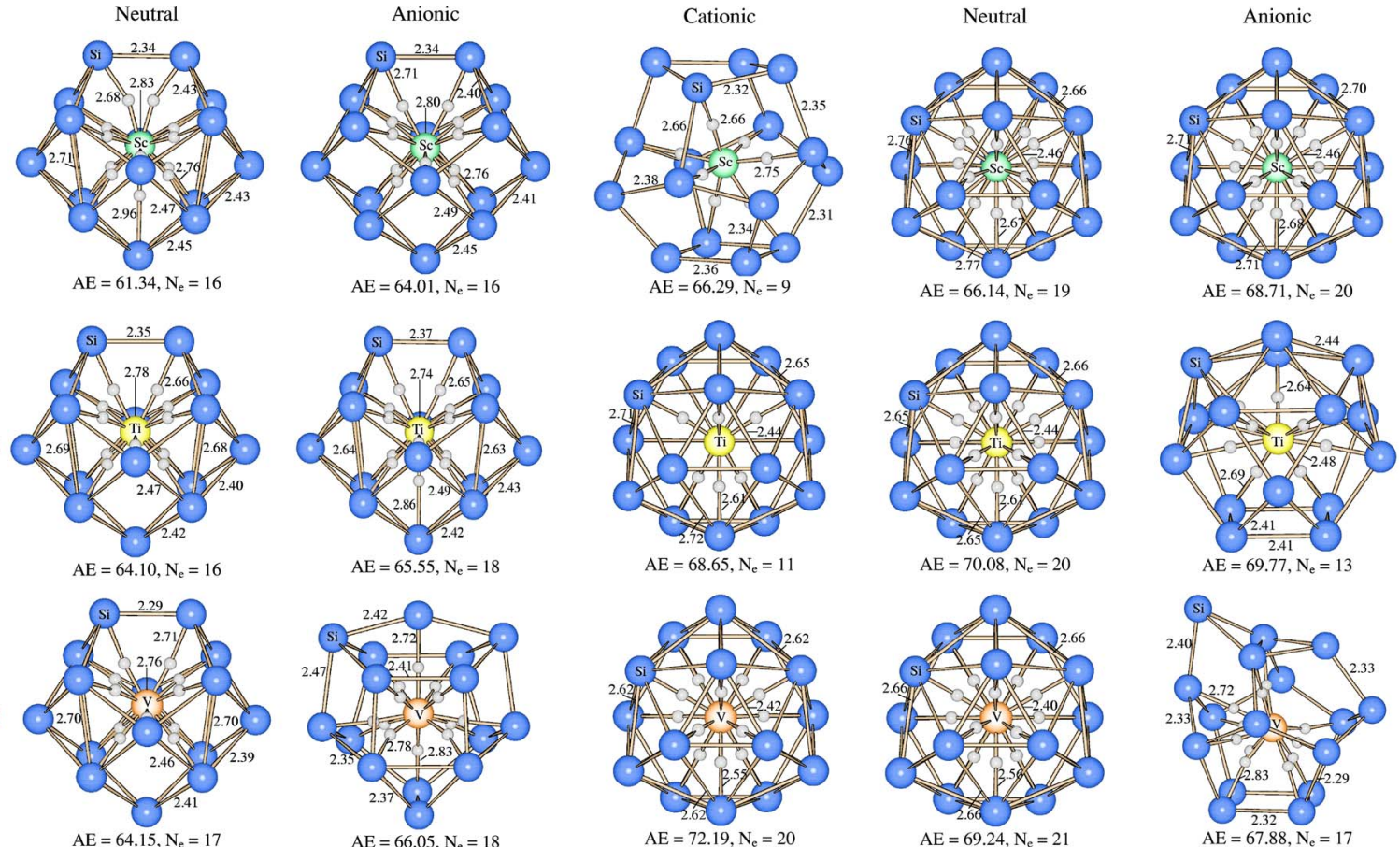

(b)
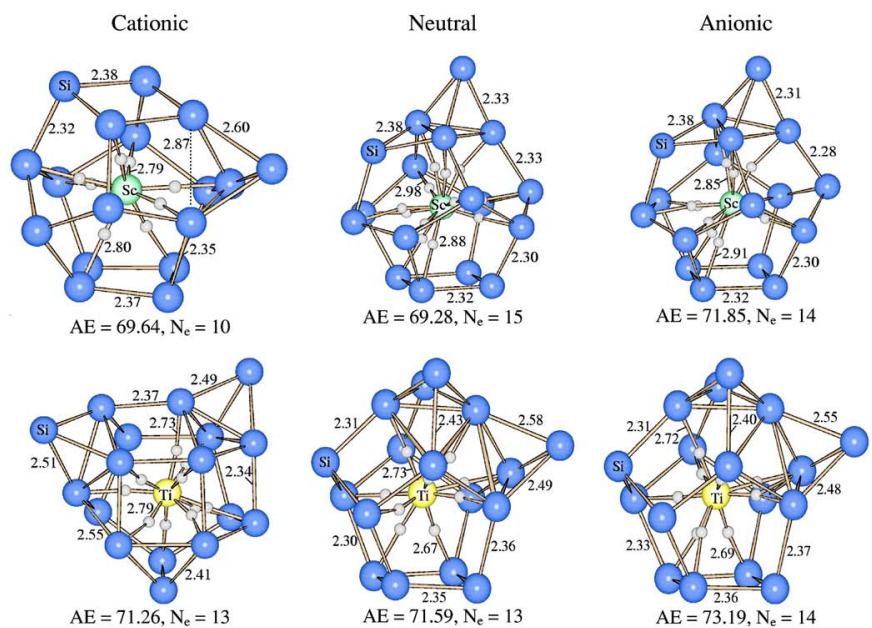

(c)
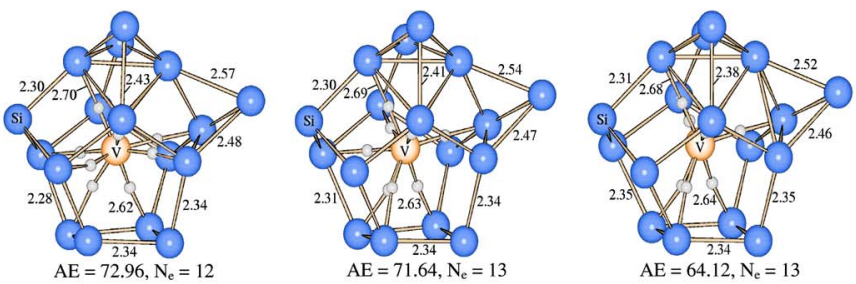

FIG. 1. (Color online) (a) Optimized geometries for the $M \mathrm{Si}_{15}(M=\mathrm{Sc}, \mathrm{Ti}, \mathrm{V})$, cationic, neutral, and anionic clusters. Atomization energies $(\mathrm{AE})$ in $\mathrm{eV}$ and number of valence electrons $\mathrm{N}_{\mathrm{e}}$. (b) Optimized geometries for the $M \mathrm{Si}_{16}(M=\mathrm{Sc}, \mathrm{Ti}, \mathrm{V})$, cationic, neutral and anionic clusters. Atomization energies (AE) in $\mathrm{eV}$ and number of valence electrons $N_{e}$. (c) Optimized geometries for the $M \mathrm{Si}_{17}$ $(M=\mathrm{Sc}, \mathrm{Ti}, \mathrm{V})$, cationic, neutral, and anionic clusters. Atomization energies (AE) in $\mathrm{eV}$ and number of valence electrons $N_{e}$.

servation rule would lead to a maximum for $\mathrm{Si}_{17} \mathrm{Ti}^{-}$and not for $\mathrm{Si}_{16} \mathrm{Ti}$, in disagreement with experimental findings. ${ }^{11}$

Examination of Figs. 1(a)-1(c) shows that clusters of the same size and composition can exhibit wide variations in geometry depending upon the charged state of the cluster. A case in point are the $M \mathrm{Si}_{16}$ clusters. $\mathrm{ScSi}_{16}{ }^{-}, \mathrm{TiSi}_{16}$, and
$\mathrm{VSi}_{16}{ }^{+}$are all characterized by most symmetric and compact Frank-Kasper polyhedrons. Note that they all correspond to a shell closing of 20 electrons. However, as the figures show, the symmetric structures undergo distortion when the number of valence electrons deviates from the shell closure. Similar distortions were also noticed in our recent work on 


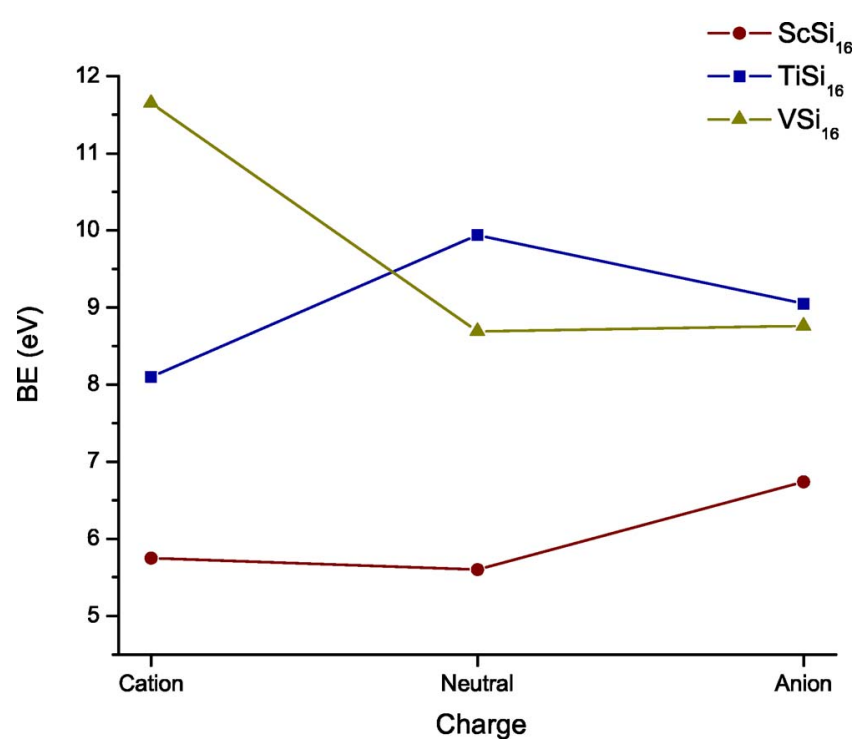

FIG. 2. (Color online) Binding energy of the transition metal to the silicon clusters, as a function of the cluster charge $(\mathrm{BE})$.

$M \mathrm{Si}_{12}$ clusters. ${ }^{9}$ We had also shown earlier for the case of alkali tetramers that open electronic shells can lead to structural distortions associated with the Jahn-Teller distortions. ${ }^{20}$

To more critically examine the electron counting rule, we now consider the change in the stability of $\mathrm{ScSi}_{16}, \mathrm{TiSi}_{16}$, and $\mathrm{VSi}_{16}$ as we change the charged state or the number of silicon atoms in the cluster. Let us begin with the change in number of electrons by changing the charged state. We calculated the energy gained as a transition metal binds to the corresponding pure $\mathrm{Si}_{n}$ cluster by calculating the binding energy (BE)

$$
\mathrm{BE}=E\left(\mathrm{Si}_{n}\right)+E(M)-E\left(M \mathrm{Si}_{n}\right) .
$$

Here $E\left(\mathrm{Si}_{n}\right)$ and $E\left(M \mathrm{Si}_{n}\right)$ are the total ground state energies of the pure silicon cluster and $M \mathrm{Si}_{n}$ cluster, respectively and again, the atomic multiplicities were chosen to enforce spin conservation. For the charged clusters, the above equation was modified to include the charge state. Here, we examined both the fragmentation channels, namely the channel where the charge is localized on the $\mathrm{Si}_{n}$ cluster or the metal atom. The lower of the two was used as BE. Figure 2 shows the variation of $\mathrm{BE}$ for the three charged states of selected clusters. Note that $\mathrm{ScSi}_{16}{ }^{-}, \mathrm{TiSi}_{16}$, and $\mathrm{VSi}_{16}{ }^{+}$are indeed the most stable species. This is in agreement with the maximum in atomization energies for these species as shown in Fig. 1(b). The clusters are grown via the accumulation of $\mathrm{Si}$ and the metal atoms and hence another important measure of stability is the progression in binding as one changes the number of Si atoms. To this end, we examined the binding energy $\mathrm{BE}$ of the metal to $\mathrm{Si}_{n}$ clusters as the number of $\mathrm{Si}$ atoms changes from 15 to 17 atoms. Figure 3 shows the resulting variation of $\mathrm{BE}$ for the $\mathrm{Si}_{n} \mathrm{Sc}^{-}, \mathrm{Si}_{n} \mathrm{Ti}$, and $\mathrm{Si}_{n} \mathrm{~V}^{+}$. Note that in each case, the maximum binding occurs for 16 $\mathrm{Si}$ atoms again reiterating the stable nature of these clusters.

Having provided theoretical evidence for the stability of $\mathrm{ScSi}_{16}{ }^{-}, \mathrm{TiSi}_{16}$, and $\mathrm{VSi}_{16}{ }^{+}$, we now come to a discussion of the geometries of the clusters. Since the configuration space

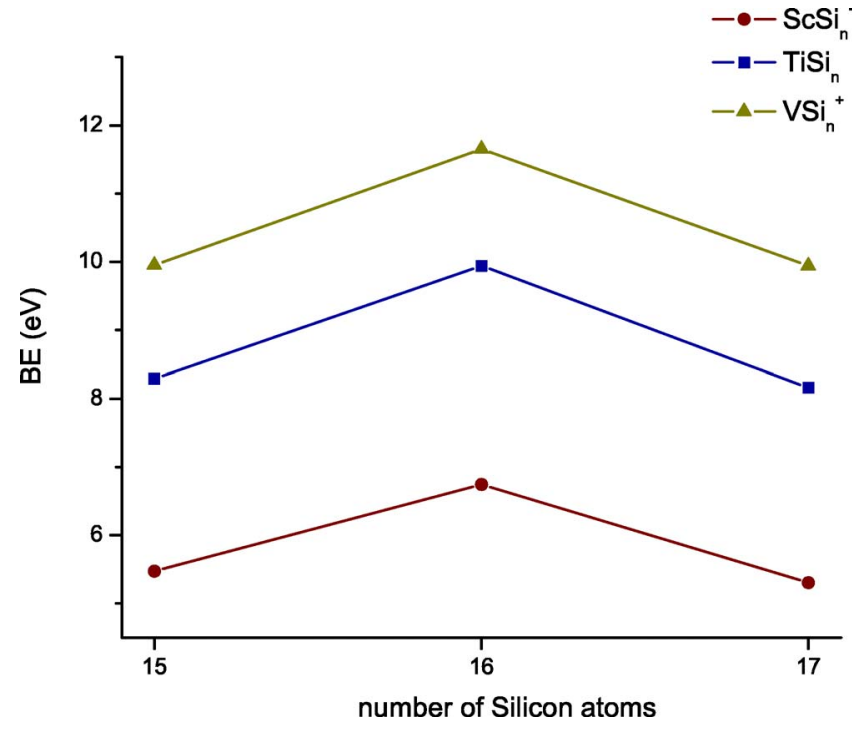

FIG. 3. (Color online) Binding energy of the transition metal to the silicon clusters, as a function of the number of silicon atoms (BE).

in small clusters is marked by local minima and since the present clusters contain more than a dozen atoms, it is virtually impossible to examine the entire configuration space. Further, the clusters are too small for microscopy and too large for spectroscopic studies. An indirect approach to probe the geometrical configurations is through synergy between theoretical studies and the negative ion photodetachment spectra. In the experiments, a selected cluster anion is crossed with a laser of fixed wavelength and the energy of the photodetached electron is measured. The difference between the energy of the photon and that of the detached electron yields the binding energy of the electron. Consider an anionic cluster that has $N$ unpaired electrons and hence a multiplicity $M=N+1$. When the electron is detached, the neutral cluster will have a spin multiplicity of $M-1$ or $M+1$ depending on whether the electron is removed from the majority or the minority state. The peaks in the photodetachment spectra corresponding to the two transitions can be compared with theoretical calculated transition energies. Here, one first determines the ground state of the anion including its spin multiplicity. One then studies the energies required to make the transitions to the neutral states with multiplicity of $M-1$ or $M+1$, with no change in the initial anionic geometry. If the calculated energies agree quantitatively with the experimental peaks, one can conclude that the spin multiplicity calculated in theory must be correct. In addition to the vertical transitions, one can compare the calculated adiabatic electron affinity with experimental values. We had earlier used ${ }^{23}$ such a procedure to identify the ground state of $\mathrm{Fe}_{3}$ and Tono et al. ${ }^{24}$ have recently used it for the ground state of $\mathrm{Cr}_{2} \mathrm{O}$.

For $\mathrm{ScSi}_{16}, \mathrm{TiSi}_{16}$, and $\mathrm{VSi}_{16}$, Koyasu et al. ${ }^{11}$ have carried out negative photodetachment spectroscopy. To make correspondence with these calculations, the theoretical vertical transitions from anions to corresponding neutrals were carried out and the results are given in Fig. 4. Table I also lists the calculated ionization potential (IP), adiabatic electron af- 
VDE
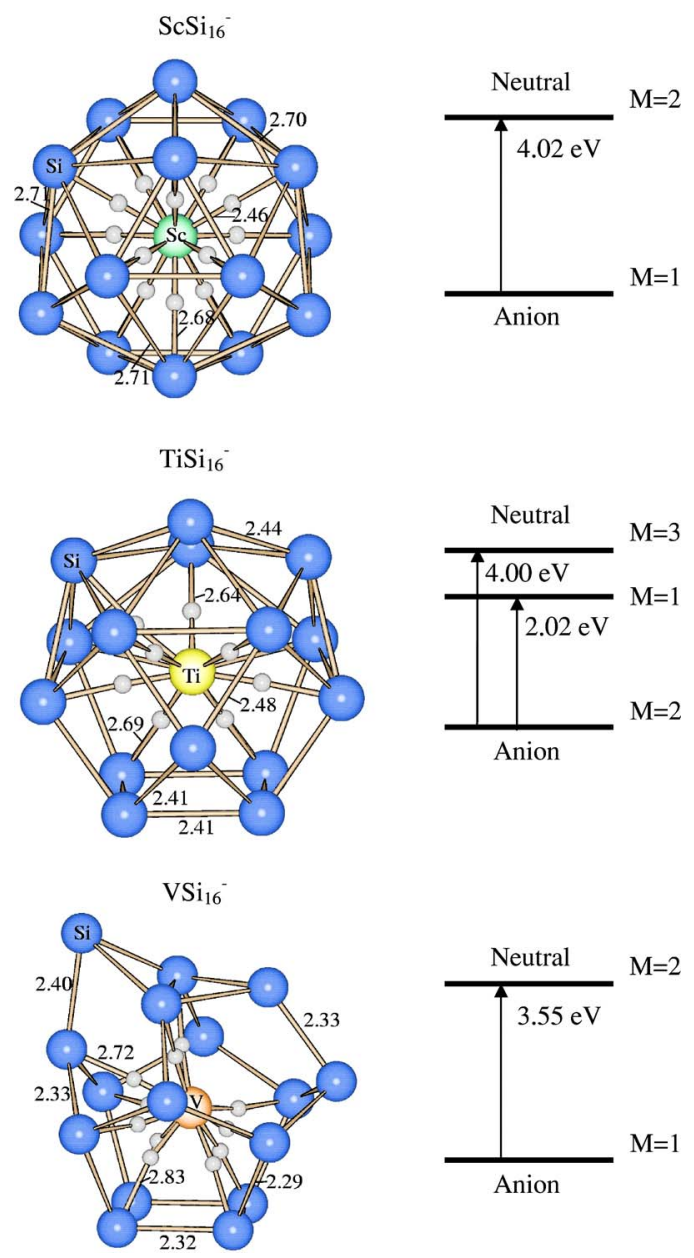

FIG. 4. (Color online) Vertical detachment energies (VDE) for the $\mathrm{ScSi}_{16}{ }^{-}, \mathrm{TiSi}_{16}{ }^{-}$, and $\mathrm{VSi}_{16}{ }^{-}$clusters.

finity (AEA), and the HOMO-LUMO gap in the three species. We start with $\mathrm{TiSi}_{16}{ }^{-}$. The experimental spectra indicate transitions around $2.4 \mathrm{eV}$ and a gap of $1.90 \mathrm{eV}$ between the first and second peak. They also find an adiabatic detachment energy (ADE) of $2.03 \mathrm{eV}$. Our calculations predict the first peak at $2.02 \mathrm{eV}$ and a gap of $1.98 \mathrm{eV}$ between the first two transitions. This gap between the two peaks is in excellent agreement. We also find an AEA of $1.91 \mathrm{eV}$ for $\mathrm{TiSi}_{16}{ }^{-}$compared to the experimental value of $2.03 \mathrm{eV}$, again showing a close agreement. We would like to add that Kawamura and co-workers also obtain similar results. ${ }^{20}$ The calculated tran-

TABLE I. Ionization potential (IP), adiabatic electron affinity (AEA), and HOMO-LUMO Gap for the $M \mathrm{Si}_{16}$ clusters.

\begin{tabular}{lccccc}
\hline \hline & & & \multicolumn{2}{c}{ HOMO-LUMO gap (eV) } \\
\cline { 4 - 6 } & Neutral $(\mathrm{eV})$ & $\begin{array}{c}\text { AEA }(\mathrm{eV}) \\
\text { Anion }\end{array}$ & Cation & Neutral & Anion \\
\hline $\mathrm{ScSi}_{16}$ & 7.01 & 3.93 & 0.76 & 0.18 & 2.26 \\
$\mathrm{TiSi}_{16}$ & 7.46 & 1.91 & 0.19 & 2.34 & 0.20 \\
$\mathrm{VSi}_{16}$ & 5.39 & 2.87 & 2.42 & 0.15 & 1.15 \\
\hline \hline
\end{tabular}

sitions for $\mathrm{ScSi}_{16}$ and $\mathrm{VSi}_{16}$ are also reproduced. For $\mathrm{ScSi}_{16}{ }^{-}$, the experimental spectra indicate an AEA of $3.41 \mathrm{eV}$ and a first peak at $4.25 \mathrm{eV}$ compared to the calculated values of 3.93 and $4.02 \mathrm{eV}$, respectively. We would like to caution that the experimental spectrum in this case is quite broad and it is difficult to assign a precise value for the AEA. Finally, for $\mathrm{VSi}_{16}{ }^{-}$, the first peak in the experimental spectra is seen around $3.5 \mathrm{eV}$ in agreement with our calculated value of $3.55 \mathrm{eV}$. The experiments also indicate a low ionization potential for $\mathrm{VSi}_{16}$. Table I shows that it is indeed the case as it has a ionization potential of $5.39 \mathrm{eV}$ comparable to that of a $\mathrm{Li}$ atom.

The key issue then is the establishment of the electron counting rules that can enable one to identify magic species. The stability of $\mathrm{ScSi}_{16}{ }^{-}, \mathrm{TiSi}_{16}$, and $\mathrm{VSi}_{16}{ }^{+}$is accounted on the premise that each $\mathrm{Si}$ donates one electron to the valence manifold. The stability of these species can then be rationalized on the basis of a 20 electron rule. However, such naive reasoning can be flawed. As an example, $\mathrm{ScSi}_{17}, \mathrm{TiSi}_{17}{ }^{+}$, and $\mathrm{VSi}_{15}$ each have 20 electrons. However, none of these is seen as a magic species in the observed mass spectra. Why then are $\mathrm{ScSi}_{16}{ }^{-}, \mathrm{TiSi}_{16}$, and $\mathrm{VSi}_{16}{ }^{+}$so special? We believe that the answer to this riddle lies in the identification of Si sites that contribute an electron to the nearly free electron gas. We propose that only those Si sites, that are bonded to a metal atom, contribute an electron. This requires a feature that enables identification of such bonds. Here we propose to use the bond critical points $(\mathrm{BCP})$ in the charge density that have been previously used to identify bonds between a pair of atoms. ${ }^{25}$ Figures $1(\mathrm{a})-1(\mathrm{c})$ show the results of such investigations where the existence of a bond is marked by the presence of a white circle (BCP). In each of the $\mathrm{ScSi}_{16}{ }^{-}, \mathrm{TiSi}_{16}$, and $\mathrm{VSi}_{16}{ }^{+}$clusters, all the $\mathrm{Si}$ atoms bond to the metal atom and hence it is proper to count one valence electron from the $\mathrm{Si}$ pool. On the other hand, in $\mathrm{ScSi}_{17}$, the analysis reveals that there are only $12 \mathrm{Si}-\mathrm{Sc}$ bonds leading to a total of 15 valence electrons. Similarly, $\mathrm{TiSi}_{17}{ }^{+}$and $\mathrm{VSi}_{15}$ have 13 and 17 valence electrons, respectively. This shows that both the geometry and the electron counting are needed to properly address the stability of metal-silicon species. We finally address the low reactivity of $\mathrm{ScSi}_{16}{ }^{-}, \mathrm{TiSi}_{16}$, and $\mathrm{VSi}_{16}{ }^{+}$with $\mathrm{H}_{2} \mathrm{O}$ seen in experiments. Our calculations reveal that these clusters have HOMO-LUMO gaps of 2.26, 2.34, and $2.42 \mathrm{eV}$, respectively. A comparison with Table I shows that these are much higher than other clusters and hence provide chemical stability to these species.

To summarize, the present investigations show that the stability of $M \mathrm{Si}_{n}$ clusters can indeed be rationalized on the basis of electron counting rules that take into account the geometrical shapes. Assuming that each Si that binds to the metal contributes one valence electron, systems where the total number of electrons is 20 do seem to exhibit enhance stability. It will be interesting to see how widespread these concepts are particularly in cases where one includes a nontransition element.

We gratefully acknowledge financial support from Department of Energy (DE-FG02-02ER46009). We are also grateful to A. W. Castleman, Jr. for discussions. 
${ }^{1}$ M. F. Jarrold, Science 252, 1085 (1995); D. E. Bergeron, P. J. Roach, A. W. Castleman, N. O. Jones, and S. N. Khanna, ibid. 307, 231 (2005).

${ }^{2}$ S. N. Khanna and P. Jena, Phys. Rev. B 51, 13705 (1995); S. N. Khanna, B. K. Rao, and P. Jena, Phys. Rev. Lett. 89, 016803 (2002).

${ }^{3}$ V. Kumar and Y. Kawazoe, Phys. Rev. Lett. 87, 045503 (2001); V. Kumar, T. M. Briere, and Y. Kawazoe, Phys. Rev. B 68, 155412 (2003).

${ }^{4}$ M. F. Jarrold and J. E. Bower, J. Chem. Phys. 96, 9180 (1992); U. Rothlisberger, W. Andreoni, and M. Parrinello, Phys. Rev. Lett. 72, 665 (1994).

${ }^{5}$ K. Jackson and B. Nellermoe, Chem. Phys. Lett. 254, 249 (1996).

${ }^{6}$ H. Hiura, T. Miyazaki, and T. Kanayama, Phys. Rev. Lett. 86, 1733 (2001).

${ }^{7}$ F. A. Cotton and G. Wilkinson, Advanced Inorganic Chemistry (Wiley, New York, 1980).

${ }^{8}$ W. D. Knight, K. Clemenger, W. A. de Heer, W. A. Saunders, M. Y. Chou, and M. L. Cohen, Phys. Rev. Lett. 52, 2141 (1984); S. Saito and S. Ohnishi, ibid. 59, 190 (1980).

${ }^{9}$ J. U. Reveles and S. N. Khanna, Phys. Rev. B 72, 165413 (2005).

${ }^{10}$ J. Lu and S. Nagase, Phys. Rev. Lett. 90, 115506 (2003).

${ }^{11}$ K. Koyasu, M. Akutsu, M. Mitsui, and A. Nakajima, J. Am. Chem. Soc. 127, 4998 (2005).

${ }^{12}$ W. Kohn and L. J. Sham, Phys. Rev. 140, A1133 (1965).

${ }^{13}$ J. P. Perdew, K. Burke, and M. Ernzerhof, Phys. Rev. Lett. 77, 3865 (1996).
${ }^{14}$ M. R. Pederson and K. A. Jackson, Phys. Rev. B 41, 7453 (1990).

${ }^{15}$ K. Jackson and M. R. Pederson, Phys. Rev. B 42, 3276 (1990).

${ }^{16}$ D. Porezag and M. R. Pederson, Phys. Rev. A 60, 2840 (1999).

${ }^{17}$ A. M. Köster, P. Calaminici, M. Casida, R. Flores-Moreno, G. Geudtner, A. Goursot, T. Heine, A. Ipatov, F. Janetzko, S. Patchkovskii, J. U. Reveles, A. Vela, and D. R. Salahub, deMon2k V. 1.08, The international deMon developers community (2005). http://www.deMon-software.com; A. M. Köster, J. U. Reveles, and J. M. del Campo, J. Chem. Phys. 121, 3417 (2004); A. M. Köster, R. Flores-Moreno, and J. U. Reveles, ibid. 121, 681 (2004).

${ }^{18}$ N. Godbout, D. R. Salahub, J. Andzelm, and E. Wimmer, Can. J. Phys. 70, 560 (1992).

${ }^{19}$ J. U. Reveles and A. M. Köster, J. Comput. Chem. 25, 1109 (2004).

${ }^{20}$ H. Kawamura, V. Kumar, and Y. Kawazoe, Phys. Rev. B 71, 075423 (2005)

${ }^{21}$ S. N. Khanna, B. K. Rao, P. Jena, and J. L. Martins, in Physics and Chemistry of Small Clusters, edited by P. Jena, B. K. Rao, and S. N. Khanna (Plenum Press, New York, 1986), p. 435.

${ }^{22}$ E. Wigner and E. E. Witmer, Z. Phys. 51, 859 (1928).

${ }^{23}$ G. L. Gutsev, S. N. Khanna, and P. Jena, Phys. Rev. B 62, 1604 (2000).

${ }^{24}$ K. Tono, A. Terakasi, T. Ohta, and T. Kondow, J. Chem. Phys. 119, 11221 (2003).

${ }^{25}$ R. F. W. Bader, Atoms in Molecules-A Quantum Theory (Oxford University Press, Oxford, 1990). 